\title{
Learning Objects in Online Education: A Systemic Approach
}

\author{
Gerardo Quiroz Vieyra \\ Mtro., Professor of the Department of Politics and \\ Culture of the Universidad Autónoma \\ Luis Fernando Muñoz González \\ $\mathrm{PhD}$, Professor of the Department of Economic \\ Production of the Universidad Autónoma
}

\section{Abstract}

In the construction of learning objects, as digital instructional material that is delivered to students, the level of learning to which they are directed must be considered first of all, according to the Bloom taxonomy or any other that is used, applicable to the e-learning, taking advantage of the digital resources that are currently available, multimedia for creation, digital repositories for storage and internet for access. Learning objects are built with pedagogical and technological elements that follow a process in themselves, subject to an educational model, instructional design, curriculum design and learning objectives as precedents, with stages very similar to those of design, construction and operation of an information system. Given their nature of digital entities, learning capsules that contain information and knowledge, the Data Quality Model of the ISO / IEC 25012: 2008 standard is also applicable, which guarantees the quality of the content and their access and availability. The creation of learning objects must also obey an educational strategy and be considered holistically in the e-learning system, that is, use a systemic approach throughout the teaching-learning process, including learning objects, considering harmonization, performance and quality in all its stages.

Keywords: cognitive computation, construction of learning objects, digital repositories, educational strategies, teaching-learning process

\section{Introduction}

Due to the COVID-19 pandemic that emerged in the city of Wuhan, China in 2019, many things have changed, and in a post-pandemic era, which is not yet in sight, much of what has changed will endure; for example, new hygiene and personal care habits, restaurant capacity, safe distances, more work at home when the nature of the activities allows it, and many others. In the educational field, there was a mandatory foray into e-learning, although not fully and completely, but through the provision of 
virtual classrooms for remote education and embryonic repositories in which teachers made materials such as presentations available to their students electronic, videos with the exposition of topics and even tutorials, also increased the use of applications for topics such as mathematics and simulation.

As a result of the unforeseen use of resources belonging to e-learning, a mixture of platforms were used, sometimes compatible but dissimilar, taking a tool from one and another from another, for example, the collaboration system of a company, with the office suite on the other, the storage of a third party and even with partial uses of LMS platforms such as Moodle.

Online education is not the electronic reproduction of face-to-face courses, nor is the provision of a classroom for remote education online education. But the urgency and many times the improvisation in the use of these technologies resulted in that the virtual classes were a reproduction of the face-to-face sessions, either synchronous or asynchronous, without taking advantage of all the resources available in digital media. Even so, this situation brought positive things, for example, the approach of teachers to these technologies, the knowledge of new tools by the students and the creation of improvised digital repositories, which do not meet security and e-learning standards, but that can be used for when you return to the classroom, either in person or hybrid.

The face-to-face courses are not designed to be taught as e-learning because they use other educational strategies and different instructional materials, most of them physical, although digital ones are beginning to proliferate, such as downloadable or searchable electronic books from digital libraries. However, current circumstances make it necessary to adjust the strategies, methods and contents to the available resources.

The product that is delivered to students in this process are learning objects, which David Wiley (2002) in his seminal work presents as follows: "The fundamental idea behind learning objects is that instructional designers can build small (relative to the size of an entire course) instructional components that can be reused a number of times in different learning contexts. Additionally, learning objects are generally understood to be digital entities deliverable over the Internet. This means that any number of people can access and use learning objects simultaneously".

\section{Methodological aspects}

\section{The teaching-learning process in online education}

Teaching-learning is a process and as such has stages, inputs and outputs. The final result depends on the alignment and performance of each stage, so each of the stages must be carefully designed, instrumented and operated.

Figure 1. Process from learning theory to teaching. 


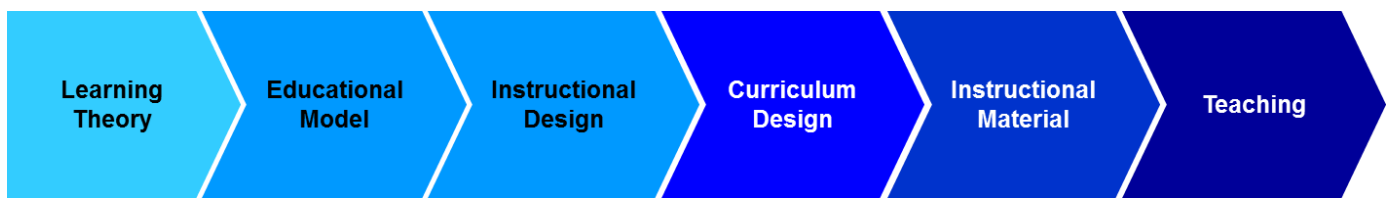

Source: self made.

In e-learning, learning objects are included in the instructional material, and are a way of presenting the learning content in digital format, with all the advantages mentioned by Wiley (2002) and his co-authors, such as simultaneous access and from anywhere you have internet access, as long as you have the permission to do so.

\section{Learning objects}

Learning objects are units of instruction, self-contained capsules with materials in various formats that develop a point on a topic. The concept derives from objectoriented programming, in which an object is a combination of variables, functions and data structures that have properties that allow their use in various ways and their reuse in more than one application, that is, they are entities that pack more than one type of elements and thus facilitate their use in other applications because, as they are self-contained, they do not depend on other elements of the program.

A learning object is defined by Barritt and Alderman (2004) as "a collection of elements that contain some information, learning activity, metadata, context and learning objective". For Joseph Frantiska Jr. (2016) "learning objects are a new way of thinking about the content of learning. Traditionally, the content comes in chunks lasting several hours. Learning objects are much smaller units of learning, typically ranging from 2 to 15 minutes".

The Wisconsin On-Line Resource Center is a successful example of a learning object system, they offer not only more than 2,500 learning objects with free access, under the Creative Commons Attribution-NonCommercial 4.0 International license, but they have also developed concepts and methodologies for its construction. In its web portal, WORC (2020) says that a learning object is "the most basic building block of a lesson or activity; searchable, usable in any learning environment; able to be grouped or to stand alone; transportable from course to course and program to program".

There are many other concepts of learning objects, from different perspectives, but all agree that they are learning units that fragment a lesson into shorter, but selfcontained pieces, so that they can be assembled together to form part of a lesson of a course or a program. This is the basic concept of use and reuse that gives flexibility and adaptability to learning objects.

The learning objects are developed by teachers who receive support from a team of specialists who align the draft created by the teacher with the instructional design, technicians who develop the objects in the selected medium, reviewers who evaluate it and the content administrator who load on the system, at a minimum. 
Figure 2. Process of instructional material as learning objects.

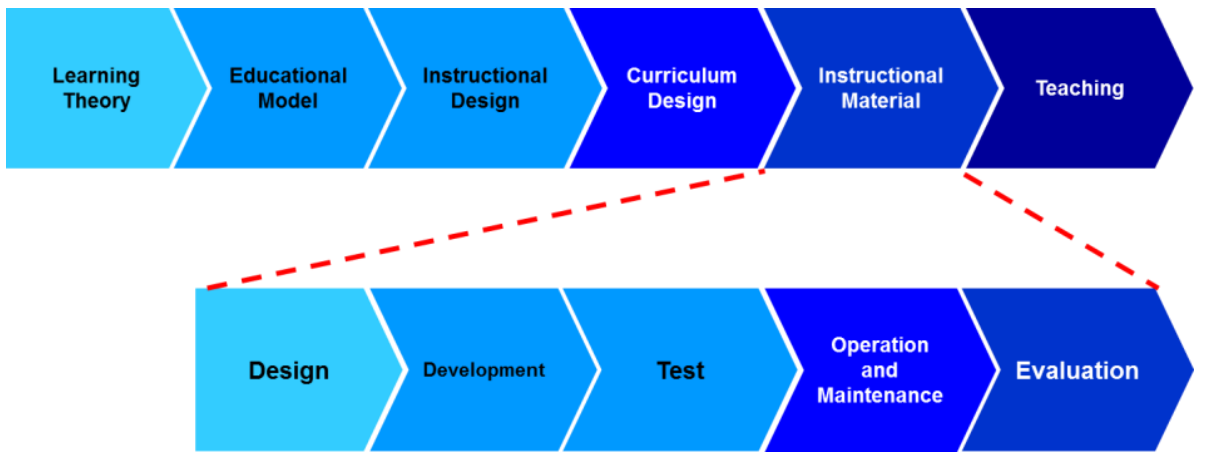

Source: self made.

Since knowledge objects are data / information capsules, it is highly recommended that they align with ISO / IEC 25012: 2008 (2008), which establishes data quality as "the degree to which the characteristics of the data satisfy established and implicit needs when used under specific conditions". This standard establishes a model of 15 characteristics for data quality: (1) accuracy, (2) completeness, (3) consistency, (4) credibility, (5) correctness, (6) accessibility, (7) compliance, (8) confidentiality, (9) efficiency, (10) precision, (11) traceability, (12) understandability, (13) availability, (14) portability and (15) recoverability. Some of these characteristics depend on the data itself $(1,2,3,4,5)$, others on the system $(13,14,15)$, and some of both $(6,7,8,9$, $10,11,12)$, but in any case they are criteria that must be taken into account in the different stages of development of learning objects.

\section{Design}

The design of learning objects is based on criteria, learning theories and establishing the learning experience and the means to achieve it, which determines the applicable methods and technologies. Diana Laurillard (2013) establishes five means for an equal number of learning experiences:

Table 1. Five main means to achieve learning experiences (modified from Laurillard, 2013, pp 90).

\begin{tabular}{|l|l|}
\hline Learning experience & Media form \\
\hline Attend, learn & Narrative \\
\hline Investigate, explore & Interactive \\
\hline Discuss, debate & Communicative \\
\hline Experiment, practice & Adaptive \\
\hline Articulate, express & Productive \\
\hline
\end{tabular}

These five forms of media remain, but methods and technology continue to change, enriching some and disappearing others, mainly due to the services offered through 
the internet such as videoconferences, streaming, collaboration systems, electronic libraries, databases, blogs, vlogs, online simulators, social networks and others.

Shantha Fernando (2009) mentions that design should focus on complying with the three "U" that Keen and Sol (2008) point out "usefulness, usability and usage", which in relation to learning objects mean that they are useful in the content of the course through the tools and methods used in it, that have usability for the people, technology and processes in which they are used and that have usage in terms of flexibility, adaptability and sustainability. Likewise, the author points out that it is not only necessary to develop the learning objects only with the correct practices, but with a new approach, which implies ways of thinking and of support for a different modeling, control and work, which are adapted to the Present and future conditions of the discipline or area of knowledge for which the learning objects are being built. This means that it is not appropriate to apply the same model to all areas or disciplines, because they can be as dissimilar as mining and oceanography, or systems engineering and medicine, requiring different approaches, resources and dynamics.

At this stage, both the pedagogical as well as the technological and operational aspects must be considered, such as the form of the medium, the technology to be used and the scope of the objects. It should be noted that a good design is the key to a good product, because that is where its architecture and engineering are established.

\section{Development}

Once the means, the strategy, the learning objectives have been determined, a preliminary sketch is built, which specialized technicians or the teacher himself, depending on the resources available, converts into a digital product using author tools.

Authoring tools are applications with which content can be created that are saved in formats compatible with e-learning standards, there are a large number of them those that are installed as complements to electronic presentation software stand out, because they combine the ease of use of the electronic presenter with the deployment and evaluation capabilities of the tool. There are also independent applications, which are not installed on top of other applications, and which include functions to create content and evaluations, saving the material created in some e-learning standard.

When the contents are going to be uploaded to an LMS or an LCMS, they must comply with some e-learning standard in order to be imported by the LMS, so if the product created with the authoring tools is not in a format supported by the LMS / LCMS, the corresponding conversion will have to be made, the same happens when using screen grabbers, video recordings, audio recordings, or any office application such as an electronic presenter, if they are to be uploaded to an LMS or LCMS the necessary 
conversion will have to be made, for which other software is needed that does "packaging" in the desired standard.

iSpring Solutions (2020) on its website describes the four prevailing standards in elearning. The oldest standard is AICC (Aviation Industry Computer-Based Training Committee) which uses the HTTP protocol, and although it has not evolved some LMS and some authoring tools still use it. The de facto standard is SCORM (Sharable Content Object Reference Model) which is currently used in two versions, 1.2 and 2004, with some differences between them. A newer standard that allows recording user "experiences", online or offline, and that has capabilities for mobile learning, social learning, offline learning, and collaborative learning is xAPI. Even more recent is cm5, launched in 2016, which brings together the benefits of SCORM and XAPI in a single standard, which means that content created under this standard can be used on mobile devices and even offline.

The choice of the e-learning standard to use will largely be dictated by the authoring tools and the LMS / LCMS used. As already mentioned, the standard in fact is SCORM, although many of the LMS / LCMS platforms can import content in SCORM or in the other standards mentioned, then the choice would be subject to the objectives set for the type of content and access to these.

\section{Storage, Access and Distribution}

Learning objects are the storage unit in e-learning systems, such as LMS (Learning Management System) and LCMS (Learning Content Management System), for which they must meet some of the standards established for them. Although the LMS and LCMS systems are the main repositories of the learning objects, external repositories can also be structured to them, for which there is software that links these external repositories with the LMS or LCMS, or simply make it accessible to the user independently of those systems.

Repositories consist of storage systems where files are deposited to be available for access. The construction and location of the repositories are also subject to strategy and evaluation. The location can be on-premise (on storage servers located within the institution), in the cloud (on a third-party storage system) or hybrid (part onpremises and part in the cloud). Determining the location of repositories takes into account performance, cost, security, availability, and all other factors that are taken into account for the location of any information system.

The creation of a repository, in addition to its location, requires careful planning of the structure, access, security, maintenance of the learning objects (updating or correcting them) and their removal when they expire. Although it only seems a technical issue, the creation and feeding of repositories also requires agreements and provision on the part of the teachers who will create the contents, an issue not easy due to the teaching style of each teacher and the recognition of the collaboration and 
authorship that make these products institutions, which can discourage their creation if they are not recognized.

Another advantage of learning objects being stored and accessible in a repository is that their reuse is facilitated, because they are seen as entities of a digital library that can be "consulted" (used or reused) by various courses and programs even by various institutions when there are agreements to do so, it is therefore very important that they meet standards in order to have interoperability and can be used by various LMS / LCMS.

In large institutions with high instructional content in the form of learning objects, a figure equivalent to the Chief Data Officer can emerge, who is responsible for the use and governance of data in a company. This administrator that we could call COLO (Chief Object Learning Officer) would be in charge of the security, availability, backup and care of the expiration of the learning objects.

\section{Thematic discussion}

\section{Evaluation}

The evaluation of learning objects is generally made on their usefulness, that is, on their content, tools and methods used and their usability for the people, technology and processes involved. Shantha Fernando (2009, pp 65) proposes the LOEM model (Learning Objects Evaluation Model) applied to usefulness, which it measures considering the specificity of the academic level, field, industry, operability and reusability. This emphasis on specificity refers to the alignment of learning objects with the educational and operational objectives of the field for which they are developed.

The evaluation of the learning objects is carried out considering both the pedagogical as well as the technological and integration aspects. For example, LOBE (Learning Object Evaluation Instrument) from the Indian Institute of Technology Bombay (2020) assesses four dimensions: content quality, pedagogical alignment, design effectiveness and technology integration, using a rubric in which they are scored on a scale of four levels the aspects to be evaluated: missing, inadequate, almost and complete.

Thus, the different models or instruments for evaluating learning objects focus on their specificity, pedagogy, technology and integration.

\section{Quality}

Joseph M. Juran, one of the three great theorists of quality management, established that quality is suitability for use, although his concept is based on production and service processes, not educational ones, it can also be considered that objects In order to be of quality they must be suitable for use, that is, in addition to fulfilling the three 
U's, they must also be capable of being reused and that they satisfy their teaching purpose as capsules of information and knowledge.

The quality of education in its different modalities has been a broad subject of study. In particular, e-learning has been studied by educational institutions, companies and governments, each establishing their own evaluation models, many of them derived or inspired by the model of the Swedish National Agency for Higher Education (2008), which considers ten factors in the evaluation of the quality of e-learning: material / contents; virtual structure / environment; communication, cooperation and interactivity; student evaluation; flexibility and adaptability; support (to students and equipment); qualification and experience of the team; institutional vision and leadership; resource allocation; and integrality, which they call the holistic aspect of the system. This model is broad and comprehensive because it considers not only the pedagogical and technical aspects, but also the institutional aspect expressed in the vision, leadership and allocation of resources that it exercises.

Other models agree that the quality of e-learning is determined by the technology, the content, the learning design, the teaching and technical team, so the greater the harmonization between them, the better the quality of the process will be achieved.

Figure 3. Main determinants of the quality of e-learning.

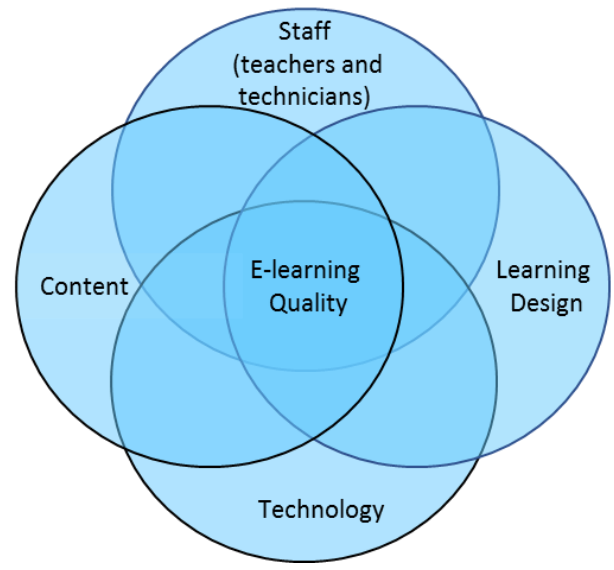

Source: self made

Undoubtedly one of the greatest demands of society is quality education, but as experts on the subject say: "quality is not measured, it is built", so quality should be one of the purposes in mind in all stages of the process of construction of learning objects.

\section{New technologies applied to learning objects}

Learning objects use multimedia resources, such as text, audio, still images and videos, and are contained in structures such as HTML, XML, JSON, etc., according to 
the standard under which they have been developed, but always with the purpose of storing them. in digital repositories, for access and deployment on personal computers or both on personal computers and on mobile devices. Although these technologies are the prevailing ones, there is already application of new technologies in learning, in different stages and with different purposes, among them machine learning and cognitive computing stand out.

Machine learning (ML) is a branch of artificial intelligence (AI) that, through classification, regression and clustering, carried out with statistical and probabilistic methods, is used to identify patterns and relationships between data. ML applied in e-learning platforms can be used to personalize courses, structuring learning objects according to the profile and progress of students, but it can also be used to analyze the learning objects themselves, that is, determine its usage patterns, evaluation results, content quality, and other aspects that provide inputs (insights) for updating, correction or replacement.

John E. Kelly III says that "Cognitive computing (CC) refers to systems that learn at scale, reason with purpose and interact with humans naturally. Rather than being explicitly programmed, they learn and reason from their interactions with us and from their experiences with their environment." IBM, a pioneer in cognitive computing with its IBM Watson system, says (Alfio Gliozzo, et al., 2017, pp 6) that the human being and cognitive computing are complementary, that while the human being is highlighted in common sense, understanding, imagination, moral, abstraction, generalization and reverie, cognitive computation is useful for locating knowledge, identifying patterns, natural language management, machine learning, elimination of biases and endless capacity, extending the cognitive capacities of the human being. Cognitive computing systems follow a decision process similar to that of humans: they observe phenomena, interpret the evidence and generate a hypothesis, evaluate the hypothesis and decide by selecting the one they consider the best option and act, all on structured data and not structured.

The characteristics of cognitive computing systems can be used in teaching-learning processes, both in teaching itself, as well as in research, problem solving and analytics. Applied to learning objects, it could make deductions about the student's journey through the course, through bots interacting with students, integrating current content relevant to the environment in the learning objects and act as the student's guide or tutor in academic matters.

\section{Conclusions and Recommendations}

Learning objects are the conjunction of pedagogical and technological aspects, which require a lot of planning and strategy. The rigor that is put into each of its stages will be reflected in the result, the student's learning. The process of construction of learning objects is very similar to that of information systems, only in this case they 
are capsules of information and knowledge, which also have in common being stored in digital repositories.

The learning objects, the elements that transmit knowledge to the student, cannot be created independently of the educational model, the instructional design, the learning objectives and the technological resources available, nor can they be created outside the institutional framework of the center educational question. A systemic, holistic approach will create the conditions for the construction of relevant and quality learning objects, which meet the expectations of use and reuse in different courses and programs.

The construction of learning objects is not the work of a single person, teacher or technician, it is the multidisciplinary work of teachers, pedagogues and technical team that, applying each of their knowledge, can result in learning capsules that cause a real impact on knowledge of the students.

New technologies, such as machine learning and cognitive computing, will make it possible to personalize learning content according to the profile and progress of students, evaluate trajectories, issue recommendations about progress and the content themselves, but nothing of this will help if the learning objects are not designed, created, tested, used, evaluated and managed with a good strategy as a basis. The idea is not to overload the process with information and communication technologies, but to provide a platform and add value to the different stages of the teaching-learning process.

In this sense, in order to achieve maximum synergy in the conjunction of all the elements set out and discussed above, it is especially advisable to establish continuous monitoring on the performance of each of the components of the system, as well as on the feedback between the teacher and the student, thus taking the necessary corrective actions in time, if applicable.

\section{References}

[1] Barritt, Ch., Alderman F.L. (2004). Creating a Reusable Learning Objects Strategy. Leveraging Information and Learning in a Knowledge Economy. John Wiley and Sons, Inc., San Francisco, CA

[2] Fernando, Shantha (2009). Designing Learning Objects for Adaptive Learning Environments. Delft University of Technology, The Netherlands.

[3] Frantiska Jr, J. (2016). Creating Reusable Learning Objects. Springer International Publishing, Switzerland. DOI 10.1007/978-3-319-32889-8

[4] Gliozzo, A, Ackerson, Ch., Bhattacharya, R., Goering, A., Jumba, A., Yeon Kim, S., Krishnamurthy, L., Lam, T., Littera, A., McIntosh Iain, Murty, S., Ribas, M. 
(2017). Building Cognitive Applications with IBM Watson Services: Volume 1, Getting Started. International Business Corporation, USA.

[5] Indian Institute of Technology Bombay (2020). LOBE: Learning Object Evaluation. Next-Education Research Lab, Educational Technology, IIT Bombay, Bombay. Retrieved November 15, 2020: http://www.et.iitb.ac.in/labs/pdf/LOBE.pdf

[6] International Organization for Standardization (2008). ISO/IEC 25012:2008. Data Quality Model. Retrieved November 18, 2020:

https://iso25000.com/index.php/en/iso-25000-standards/iso-25012.

[7] iSpring Solutions (2020). eLearning Standards Comparison: AICC vs SCORM vs XAPI vs cmi5. https://www.ispringsolutions.com/blog/elearningstandards

[8] Keen, G.W., Sol, H.G. (2008). Decision Enhancement Services: Rehearsing the Future for Decisions That Matter. Delft University Press, The Netherlands.

[9] Kelly, J. III (2015). Computing, cognition and the future of knowing: How humans and machines are forging a new age of understanding. IBM Research, Albany, NY. Retrieved November 9, 2020: https://www.research.ibm.com/software/IBMResearch/multimedia/Comp uting_Cognition_WhitePaper.pdf

[10] Laurillard, D. (2013). Rethinking University Teaching: A Conversational Framework for the Effective use of Learning Technologies. 2nd. Ed. RoutledgeFalmer, Nueva York, NY

[11] Swedish National Agency for Higher Education (2008). E-learning Quality. Aspects and criteria for evaluation of e-learning in higher education. Report 2008:11 R. Retrieved August 2, 2020: http://www.divaportal.org/smash/get/diva2:283764/FULLTEXT01.pdf

[12] Wiley, D. Editor (2002). The instructional Use of Learning Objects. Agency for Instructional Technology and Association for Educational Communications \& Technology, Bloomington, Indiana.

[13] Wisconsin On-line Resource Center (2020). "About Learning Objects". https://www.wisc-online.com/about-learning-objects. Retrieved: November 11, 2020. 